\title{
Optimal Fitting of High-Frequency Cable Model Parameters by Applying Evolutionary Algorithms
}

\author{
S. Bogarra ${ }^{1}$, J.-R. Riba ${ }^{*}$, V. Sala-Caselles ${ }^{2}$, A. Garcia ${ }^{1}$ \\ ${ }^{1}$ Electrical Engineering Department, Universitat Politècnica de Catalunya, Rambla Sant Nebridi 22, 08222 \\ Terrassa \\ ${ }^{2}$ Electronics Engineering Department, Universitat Politècnica de Catalunya, Rambla Sant Nebridi 22, \\ 08222 Terrassa \\ *Corresponding author. E-mail: riba@ee.upc.edu
}

\begin{abstract}
Due to the widespread use of electronic power converters, low-voltage high-frequency cable models are being increasingly applied in industry, automobile or aeronautics applications among others. It is known that depending on switching frequency, cable configuration and length, transient overvoltage effects comprising a wide frequency range from dc up to several tens of $\mathrm{MHz}$ can appear. However, to accurately reproduce the wide-band frequency response, such models often require the use of ladder networks, thus being necessary to adjust the values of a relatively large number of $R, L$ and $C$ components, which is a complex task. This paper is focused to solve this problem, which is done by applying an iterative genetic algorithm (IGA) optimization approach. From a set of experimental short circuit and open circuit tests the high-frequency cable model of a given cable configuration is obtained, whose parameters are fitted by means of the proposed IGA-based method. Finally, the accuracy of the model obtained is validated experimentally by comparing the frequency-domain and time-domain responses through overvoltage predictions of different samples of the analyzed cable.
\end{abstract}

Keywords-Model optimization, genetic algorithm, parameter estimation, cable model, frequency response.

\section{INTRODUCTION}

Conducted disturbances are boosted in power cable systems fed by switching PWM-modulated power electronic converters [1]. The uninterrupted and fast switching of PWM power converters tends to induce significant transient surges containing steep-fronted impulses that depend on the technology of the semiconductor switches of the electronic power converter. PWM-modulated power converters using IGBTs (insulated gate bipolar transistors) are characterized by short pulse rise times of 100-400 ns [2] and fast switching frequencies in the $2-20 \mathrm{kHz}$ frequency range [3]. Under high switching speed and having substantial distance between the drive and the load, significant voltage overshoot could happen on the load side. The high-frequency performance of power cables intended for PWM (pulse width modulation) 
applications is of a paramount importance due to the appearance of phenomena such as skin and proximity effects, transient overvoltage or conducted electromagnetic disturbances. To accurately predict such effects, a high-frequency cable model is required. Improved cable models must include low- and high-frequency effects, and must be able to reproduce fast overvoltage transients. Therefore, the development of power systems models to predict electromagnetic transients is gaining importance in view of the numerous studies for both ac and dc power systems [4-6]. The combined effect of fast rising and falling times of voltage pulses, high switching frequencies, and cable length may result in serious disturbances [7] that can overstress insulation systems at the load and inverter sides. Therefore, common-mode currents of high frequency are induced as well as $\mathrm{d} v / \mathrm{d} t$ overvoltage transients at the load terminal, which are boosted by the impedance mismatch between cable and load, which can damage insulation systems and reduce cable-load performance. This effect has been studied by several authors, especially in induction motor drive systems fed by long cables [8-10]. The sudden overvoltage effects in PWM-modulated drive systems comprise a wide range of frequencies since they include the switching harmonics [11] and the resonance frequency of the cable [7]. Depending on cable length they can range from dc up to the $\mathrm{MHz}$ range [10,11]. Therefore high-frequency models of lengthy cables are appealing to accurately analyze these electromagnetic effects in systems fed by electronic power converters, since they allow reducing design time, costs [1] and failure occurrence. Such models must take into account both the distributed nature of the cable and the frequency dependence of the effects derived [11].

Different cable models have been proposed to reproduce both the frequency and temporal behavior. The telegrapher's equations, which are a set of partial differential equations that allow describing the current and voltage on a transmission line with respect to time and line distance, can be transformed into a set of difference equations $[6,8,9,12]$ or into a set of ordinary differential equations (macromodel) through some kind of discretization process $[1,7,10,11,13,14,15]$. The model dealt with in this work is based on the last transformation despite the inconvenience in computational burden, although this drawback is minimized thanks to the use of existing computers.

Cable models may be roughly classified into different categories, that is, lumped parameter or distributed parameter models $[1,5,6,7,12,14,15]$ and constant parameter or by frequency-dependent parameter models. Cable models with distributed parameters can be more accurate than lumped parameter models depending on cable length and signal frequency, although the later ones allow a better physical interpretation of the problem while simplifying the mathematical complexity, so they can be easily integrated in any electromagnetic transients program [12]. Constant-parameter cable models [5] are less accurate than cable models taking into account frequency-dependent features $[1,6,7,8,9,10,11,12,13$, $14,15]$ to simulate transient effects, however they are computationally faster, although CPU requirements 
of the last models are not excessive for current computers. Frequency-dependent cable models are usually developed in the frequency-domain, so time-domain results are obtained by applying numerical transformations. Cable models based on lumped parameters are developed in the time domain, but the frequency-dependent features can be included in the model by using cascade of cells with lumped parameters $[8,9,10,11,13,15]$, the accuracy of the equivalent circuit depending on the number of elements $(R, L$ and $C)$ in each cell, the number of cells, the length of the cable and the analyzed frequency range [13]. Lumped parameter cable models can be developed directly in the time-domain for transient simulations. This is an advantage of such cable models since most power components are modeled in the time domain, so a cable model based on the electric circuit approach, using cascade of cells with lumped parameters is applied in this work.

Parametric cable models can be based on measurements $[1,5,7,8,9,13,14,15,23,32]$ or can be calculated as a function of the cable structure and the physical characteristics of the cables by finiteelement analysis [2,14] or by applying specific equations [10,11,13]. The last approach results in certain inaccuracies because of the applied approximations whereas parametric models adjusted by means of finiteelement analysis require large preparation and simulation times, so measurement-based parametric models outperform the other options. However, cable parameters estimation from measurements is not a simple task, but the models can be accurate for a wide frequency range even under the presence of resonance phenomena, so this option is applied in this work. Models with a limited number of elements facilitate their fitting $[8,9,15]$, but in this work a methodology is established for any number of elements in each cell and for a wide frequency range and under resonance effects. Parameters estimation usually requires the use of complex algorithms [1, 10, 13], so from a practical standpoint this paper proposes to use a GA-based approach to simplify the parameter estimation and fitting process [7].

To accurately reproduce transient overvoltage effects, a high-frequency model of both, the cable and the load is required. The high-frequency cable model must include effects such as the eddy currents (skin and proximity effects) and dielectric losses. To this end, lumped parameter models are appealing since they allow replicating the temporal and frequency response of spatially distributed physical systems such as cables by constructing a topology composed of different discrete entities or cells connected in series [11]. It is a recognized fact that such models can accurately predict the overvoltage transients when selecting an appropriate number of cells [14]. These models can include $R$ - $L$ ladder networks to simulate highfrequency eddy currents effects [7] and $G$-C ladder networks to model capacitive effects to ground [5]. High-frequency lumped parameter models have also been successfully applied to other electrical systems such as to simulate the high-frequency behavior of power transformers [15,16], ac electrical motors $[14,17]$ or servo drives [18], sometimes in combination with finite element models to determine the values of the 
constitutive parameters [19]. A possible approach to solve the whole electrical network composed of the discrete cells, is the transmission-line modelling (TLM) technique [8] which uses discrete models of the components involved and allows accurately reproduce the overvoltage transients. The TLM approach provides an accurate alternative to the state equation formulation while avoiding to solve the associated differential equations $[20,21]$.

This paper focuses on the optimal fitting of the parameters included in wide-band low-voltage cable models fed by switching power converters, which are of very interest in industry, automobile or aeronautics applications among others. Therefore the high frequency effects due to the switching power converter are dominant and greatly influence cable behavior and response.

This paper is focused to accurately adjust the parameters involved in an improved lumped cable model for multi-conductor cables of arbitrary length. This model is intended to estimate overvoltage transients by taking into account frequency effects when the cable system is fed by a PWM-modulated switching power converter. The cable model applied in this paper is similar to that proposed in [22] but using only one cell per meter of cable length instead of 32 , thus greatly reducing the computational requirements and the simulation time without any significant effect on model accuracy, as proved in Section IV. Each single cell includes a longitudinal and a transversal impedance in a ladder circuit configuration. The ladder equivalent circuit of the longitudinal impedance allows taking into account both the skin and proximity effects, whereas the ladder equivalent circuit of the transversal impedance allows considering the dielectric losses. Genetic algorithms (GA) have been widely applied in optimization problems in many disciplines $[23,24]$ including the optimal estimation of transmission line parameters [25], transformers parameters [16,26] or impulse generators [27] among others. In [28] nonlinear transfer functions were approximated by applying a GA approach, by which the optimal values of the expansion coefficients were found, thus providing more accurate results than the classical Chebyshev polynomial approximation. Sales et al. [29] developed a multi-objective GA-based method for line topology identification, using either one-port or two-port experimental data. In [7] a frequency-domain cable model was adjusted by applying a GA optimization procedure to determine the parameters of the impulse propagation function in the Laplace domain. This paper proposes a new fitting procedure to determine the optimal values of the parameters constituting each single cell of the ladder network used to model the cable. This is a nonlinear optimization problem with a large search space [30] that requires the use of efficient search algorithms to reach an accurate solution while maintaining the computational burden as low as possible. The proposed fitting method is based on a genetic algorithm (GA) approach to select the optimal values of such parameters to accurately reproduce both the frequency-domain and time-domain response of the whole cable. In this paper an interactive GA algorithm to determine the optimal values of the parameters of a single cell is presented. 
The suggested method is straightforward and does not require additional simulation or learning software tools such as FEM (finite element method) or APLAC, as required by other approaches [22].

It must be pointed out that although the IGA-based approach proposed in this paper has been applied to adjust the values of the parameters of the cable model, it can be applied to many other problems such as fitting of parameters in filters or in high-frequency models of electrical machines such as transformers, motors and generators among others.

\section{THE CABLE MODEL}

The cable model detailed in this paper tries to balance two compromises, that is, model accuracy and complexity, the last one being directly related to the computational burden. To achieve these goals, some assumptions have been done. For example the distances among the three cables are assumed to be identical (the symmetry simplifies cable modeling) and the screen has been modelled as a perfect conductor. To accurately reproduce the transient behavior of multi-wire screened cables fed by switching power converters, they must be represented by complex electric models taking into account different capacitive and inductive effects that arise at high frequency operation [31].

In this paper it is assumed that the lossy aspect of insulators presenting charge flow can be modelled by a conductance $G$ and the effects of the displacement current are assumed to be very small [32].

This work deals with the screened three-wire Exzhellent RC4Z1-K (AS) 0.6/1.0 kV cable from GeneralCable, which is schematically represented in Fig. 1.

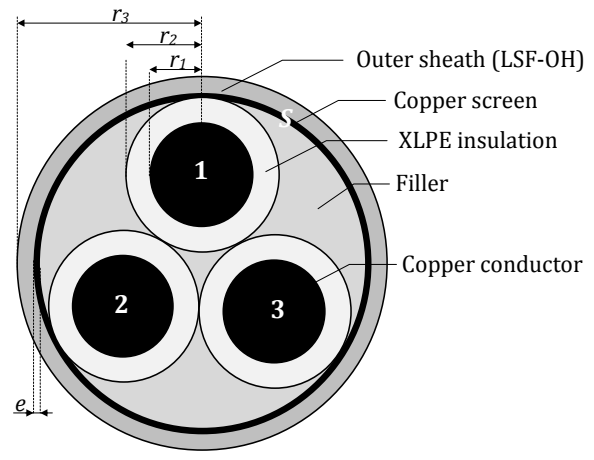

Fig. 1. Geometry of the three-wire screened cable analyzed in this work.

The cable model assumes complete symmetry, and the screen is modeled as an ideal conductor. The comparison between simulation results and experimental measurements will prove that these assumptions are justified. Main parameters of the analyzed cable are summarized in Table I.

TABLE I. PARAMETERS OF THE RC4Z1-K

(AS) THREE-WIRE SCREENED CABLE

\begin{tabular}{cc}
\hline Cable parameter & Value \\
\hline$r_{1}$ & $1.5 \mathrm{~mm}$ \\
\hline
\end{tabular}




\begin{tabular}{cc}
\hline$r_{2}$ & $2.2 \mathrm{~mm}$ \\
$r_{3}$ & $7.1 \mathrm{~mm}$ \\
$e$ & $0.16 \mathrm{~mm}$ \\
$\rho$ & $1.98 \cdot 10^{-8} \Omega / \mathrm{m}$ \\
\hline
\end{tabular}

\subsection{High-frequency multi-cell cable model}

This paper assumes a high-frequency multi-cell cable model composed of $n$ identical cells connected in series, each cell representing a length of 1 meter, as shown in Fig. 2.

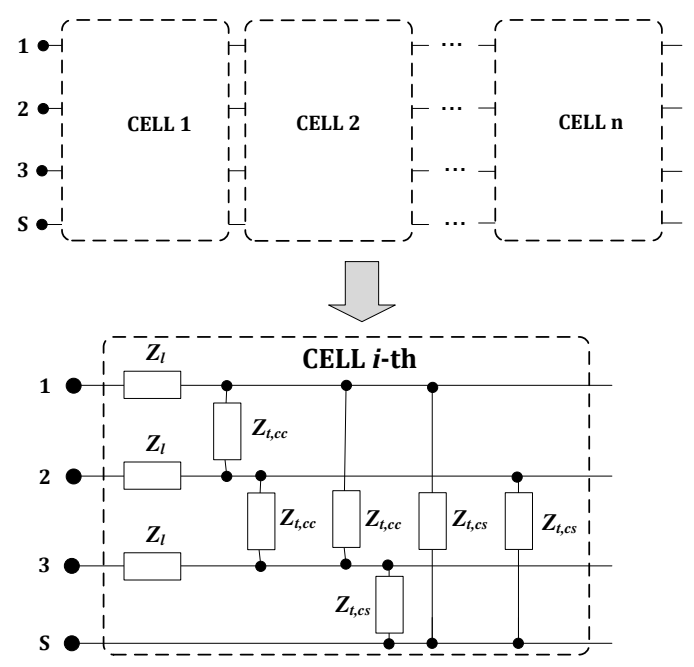

Fig. 2. Multi-cell model of the three-wire screened cable

Since all cells are considered to be identical, the values of their constitutive $R, L$ and $C$ components must also be identical. Whereas $Z_{l}=R_{l}+\mathrm{j} \cdot X_{l}$ is the complex longitudinal impedance of a single wire, $Z_{t, c c}=R_{t, c c}$ - $\mathrm{j} \cdot X_{t, c c}$ is the transversal impedance between conductor-conductor and $Z_{t, c s}=R_{t, c s}-\mathrm{j} \cdot X_{t, c s}$ is the complex transversal impedance between conductor-screen, these impedances being represented in Fig. 3.
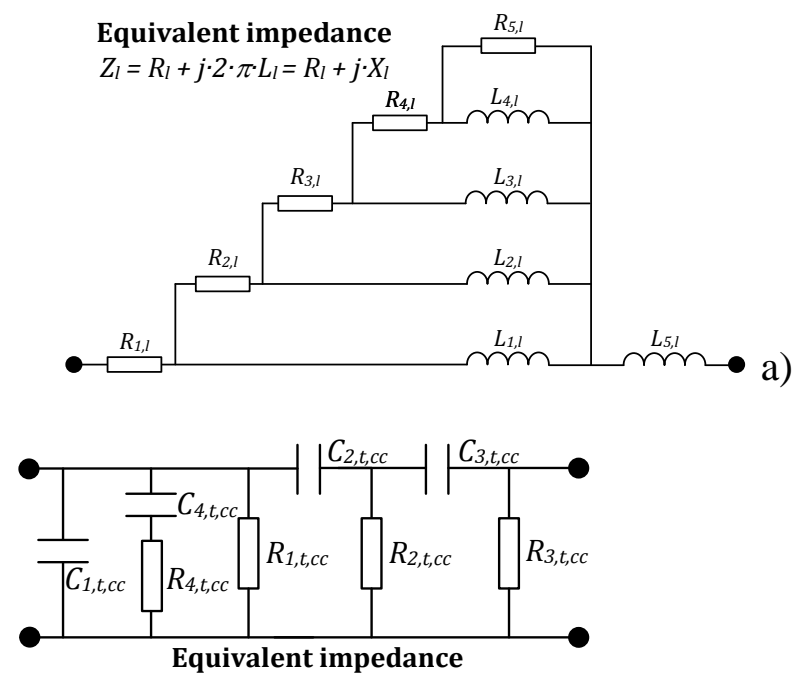

$Z_{t, c c}=R_{t, c c}-j /\left(2 \cdot \pi \cdot C_{t, c c}=R_{t, c c}-j \cdot X_{t, c c}\right.$ 


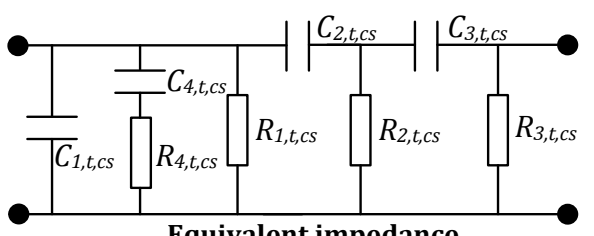

$Z_{t, c s}=R_{t, c s}-j /\left(2 \cdot \pi \cdot C_{t, c s}=R_{t, c s}-j \cdot X_{t, c s}\right.$

c)

Fig. 3. Longitudinal and transversal impedances of the single-cell model. a) Longitudinal impedance, $Z_{l}$. b) Transversal impedance between two conductors, $Z_{t, c c}$. c) Transversal impedance between conductor and screen $Z_{t, c s}$.

According to Fig. 3, the components of $Z_{l}=R_{l}+j \cdot 2 \cdot \pi \cdot f \cdot L_{l}, Z_{t, c c}=R_{t, c c}-j /\left(2 \cdot \pi \cdot f \cdot C_{t, c c}\right)$ and $Z_{t, c s}=R_{t, c s}$ $j /\left(2 \cdot \pi \cdot f \cdot C_{t, c s}\right)$ are,

$\left\{\begin{array}{l}Z_{l}=g_{1}\left(R_{1, l}, R_{2, l}, R_{3, l}, R_{4, l}, R_{5, l}, L_{1, l}, L_{2, l}, L_{3, l}, L_{4, l}, L_{5, l}, f\right)=R_{l}(f)+j \cdot X_{l}(f) \\ Z_{t, c c}=g_{2}\left(R_{1, t, c c}, R_{2, t, c c}, R_{3, t, c c}, R_{4, t, c c}, C_{1, t, c c}, C_{2, t, c c}, C_{3, t, c c}, C_{4, t, c c}, f\right)=R_{t, c c}(f)-j \cdot X_{t, c c}(f) \\ Z_{t, c s}=g_{3}\left(R_{1, t, c s}, R_{2, t, c s}, R_{3, t, c s}, R_{4, t, c s}, C_{1, t, c s}, C_{2, t, c s}, C_{3, t, c s}, C_{4, t, c s}, f\right)=R_{t, c s}(f)-j \cdot X_{t, c s}(f)\end{array}\right.$

where $g_{1}, g_{2}$ and $g_{3}$ are different functions of the parameters between brackets, $f$ is the electrical frequency and $G_{t, c c}=1 / R_{t, c c}$ and $G_{t, c s}=1 / R_{t, c s}$.

To determine the $R, L$ and $C$ parameters of the cell model in Figs. 2 and 3, four experimental tests are required [33], that is two open circuit tests and two short circuit tests to determine the frequency response in the $40 \mathrm{~Hz}-30 \mathrm{MHz}$ interval of the cable by using a wide-band high-precision impedance analyzer. Such tests are summarized in Figs. 4.

Four tests were applied to two cable samples of 0.1 meter and $1 \mathrm{~m}$ length, respectively, for determining the parameters and having satisfactory high frequency sensitivity. Therefore, two short circuit tests with 2 or 3 conductors in short circuit (SC2 and SC3, respectively), and two open circuit tests, with 2 or 3 conductors in open circuit (OC2 and OC3).
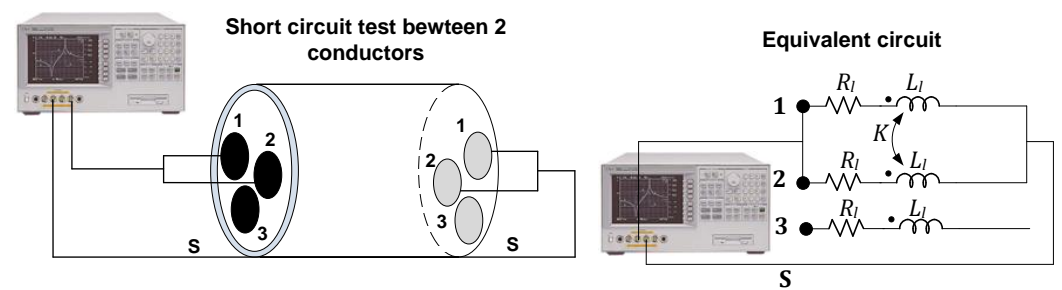

a)
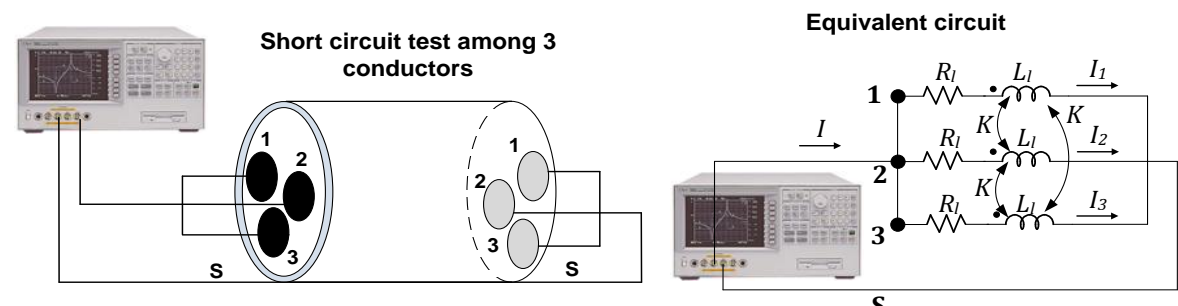

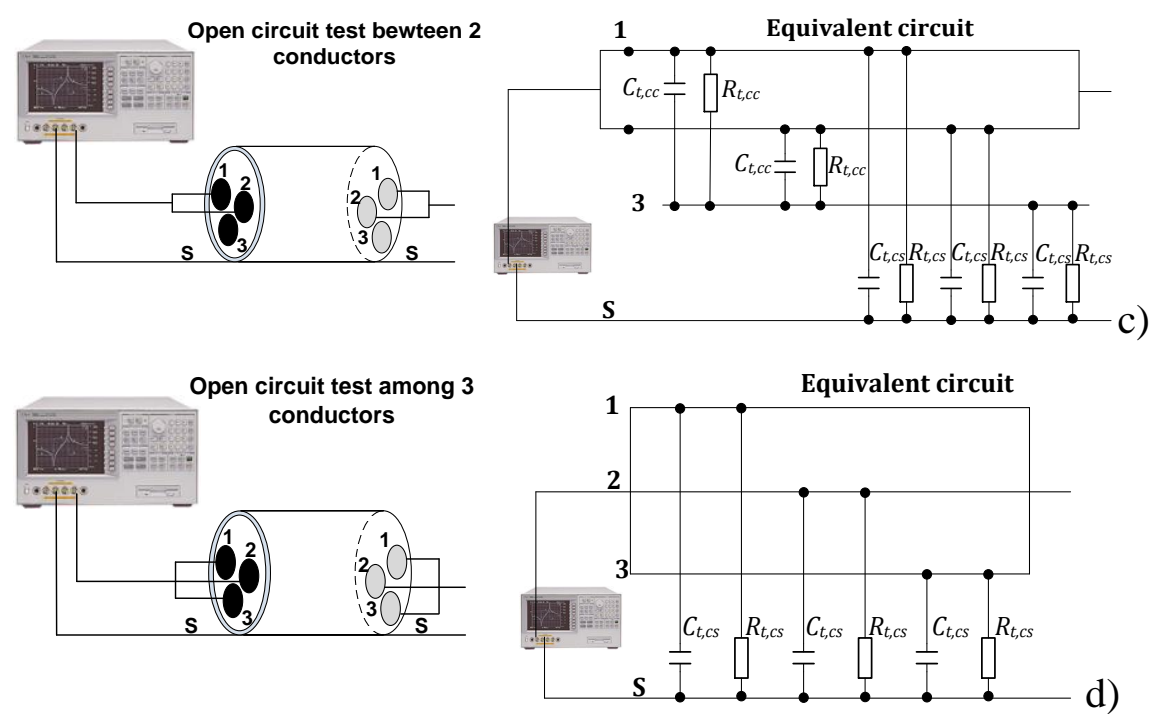

Fig. 4. Schematics of the fours tests conducted to determine the single-cell parameters using the impedance analyzer. a) Short circuit test between two conductors (SC2). b) Short circuit test among three conductors (SC3). c) Open circuit test between two conductors (OC2). d) Short circuit test among three conductors (OC3).

From the equivalent circuits of the four tests, the following set of equations arise [33], from which the parameters required to generate the cell model in Fig. 3 are calculated by solving the equations system in (2),

$$
\left\{\begin{array}{l}
L_{m, S C 2}=\left(L_{l}+M\right) / 2 \\
R_{m, S C 2}=R_{l} / 2 \\
L_{m, S C 3}=\left(L_{l}+M\right) / 3 \\
R_{m, S C 3}=R_{l} / 3 \\
C_{m, O C 2}=\left(2 C_{t, c c} \cdot C_{t, c s}\right) /\left(2 C_{t, c c}+C_{t, c s}\right)+2 C_{t, c s} \\
G_{m, O C 2}=2 G_{t, c c} \cdot G_{t, c s} /\left(2 G_{t, c c}+G_{t, c s}\right)+2 G_{t, c s} \\
C_{m, O C 3}=3 C_{t, c s} \\
G_{m, O C 3}=3 G_{t, c s}
\end{array}\right.
$$

where $G=1 / R$ and subscript $m$ means a measured value.

Fig. 5 summarizes the methodology applied to determine the cell parameters $L_{l}, R_{l}, C_{t, c c}, C_{t, c s}, R_{t, c c}$ and $R_{t, c s}$ in the $40 \mathrm{~Hz}-30 \mathrm{MHz}$ frequency range. From the steps visualized in Fig. 5, the parameter values of the cell model shown in Fig. 3 are obtained to match the measured frequency response of the cable of $1 \mathrm{~m}$ length. Finally, if a $n$-meter cable has to be simulated, the model reproducing the behavior of such a cable will require $n$ identical cells connected in series as shown in Fig. 2. 


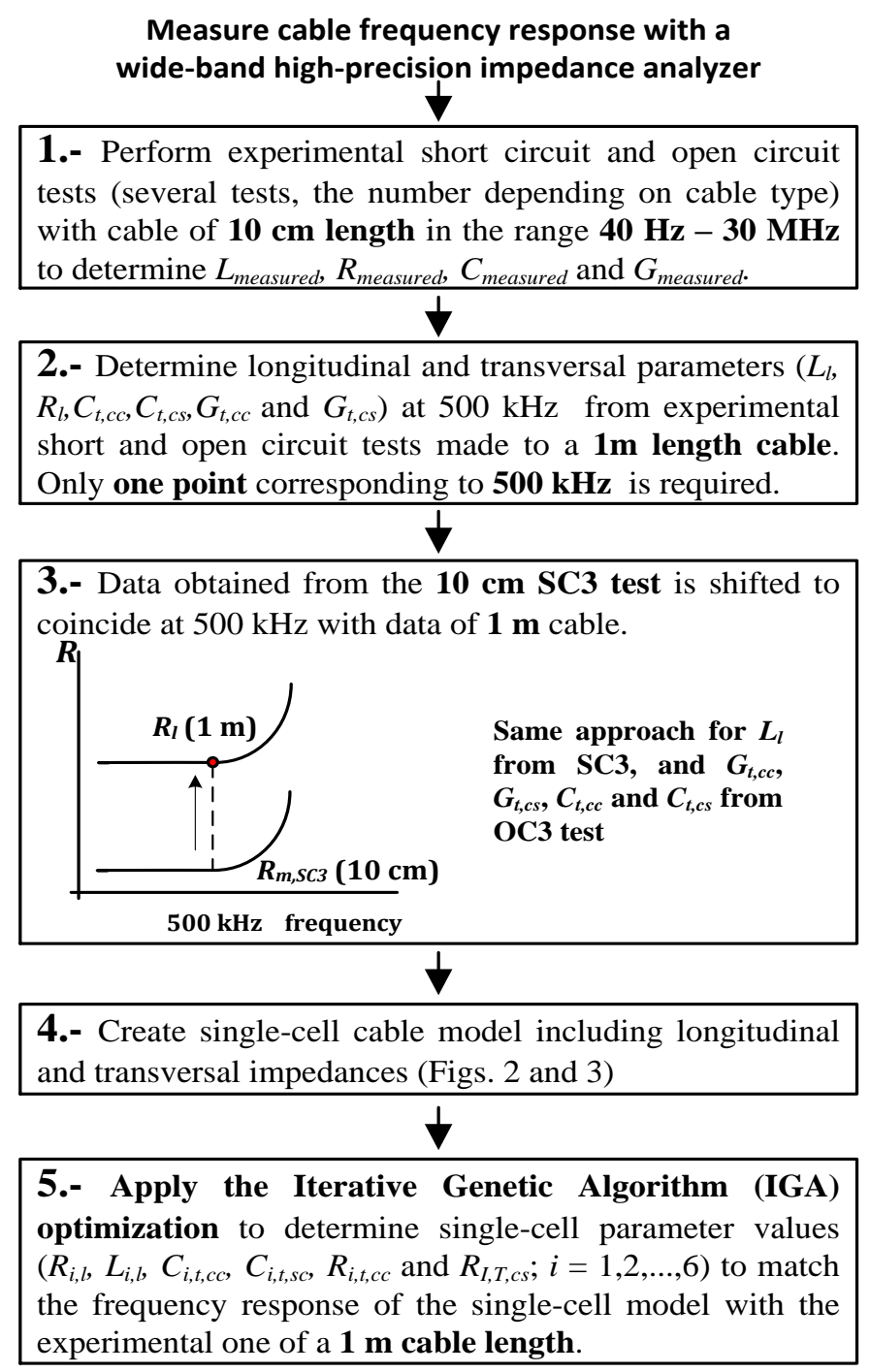

Fig. 5. Five-step methodology applied to determine the cell parameters $L_{l}, R_{l}, C_{t, c c}, C_{t, c s}, G_{t, c c}$ and $G_{t, c s}$ in the $40 \mathrm{~Hz}-30 \mathrm{MHz}$ frequency range.

As shown in Fig. 5, the frequency response of parameters $R_{l}, L_{l}$ obtained from the experimental short circuit test SC3, and $G_{t, c c}, G_{t, c s}, C_{t, c c}$ and $C_{t, c s}$ obtained from the experimental open circuit test OC3 are generated for both $10 \mathrm{~cm}$ and $1 \mathrm{~m}$ cable lengths. The shape of the measured frequency response $R_{m, S C 3}(10 \mathrm{~cm})$ determined from the $10 \mathrm{~cm}$ length is considered to be the correct one since the resonance peaks are located beyond the analyzed frequency range. Therefore the frequency response curve of the $10 \mathrm{~cm}$ cable length $R_{m, S C 3}(10 \mathrm{~cm})$ is shifted to coincide with the $500 \mathrm{kHz}$ point of the $1 \mathrm{~m}$ curve $R_{m, S C 3}(1 \mathrm{~m})$ since the point of $500 \mathrm{kHz}$ is far from the resonance peaks, thus obtaining $R_{l}(1 \mathrm{~m})$ as displayed in step 3 . Although this explanation refers to $R_{l}$ it also applies to the other parameters $L_{l}, G_{t, c c}, G_{t, c s}, C_{t, c c}$ and $C_{t, c s}$.

\section{THE ITERATIVE GENETIC ALGORITHM (IGA) APPROACH}

Genetic algorithms (GAs) are recognized as an effective tool to solve a wide diversity of optimization problems. These mathematical methods are suitable to solve both unconstrained and constrained 
optimization problems [34] in which the objective function to be minimized can be either single- or multiobjective. GAs offer several advantages compared to other optimization methods, since they do not require an initial approximation of the solution, can deal with non-linear and discontinuous objective functions and allow seeking for globally optimal solutions [30]. GAs apply the natural selection concept, that is, the survival of the most adapted or fitted specimens. The specimens generating a most fitted outcome have a high reproduction likelihood but those generating least fitted solutions have a lower chance [34]. GAs perform a heuristic iterative search so that at each iteration step they randomly select a set of specimens or individuals from the existing population, representing the chromosomes. After some iterations, that is, several generations later, the solution is supposed evolving towards an optimum. The individuals producing a local optimal are chosen at each step and become the parents of the next generation of offspring specimens. This process is repeated until achieving the best fitted individuals' population, that is, the population producing the optimal solution.

GAs apply different mathematical operations to emulate the mechanisms of genetic reproduction such as the selection of the fittest individuals, which are the parents of the next generation of offspring individuals, the crossover operation that merges the solutions corresponding to two parents to create the next generation of children solutions, and the mutation operation that produces random modifications to children and parents solutions to evolve towards an optimal solution.

In this paper a single-objective GA approach is proposed, in which the objective function to be minimized is the per unit error calculated as,

$\varepsilon=\sum_{i=1}^{20}\left[R_{c a l}(i) / R_{m}(i)-1\right]^{2}+\sum_{i=1}^{20}\left[X_{c a l}(i) / X_{m}(i)-1\right]^{2}$

the subscript $\mathrm{cal}$ denoting the parameter value calculated at each optimization step and the subscript $m$ corresponding to the measured or experimental value of the parameter. Since the impedance analyzer provides pairs of $R_{m}-f$ and $X_{m}-f$ vectors with hundreds of components, $f$ being the electrical frequency, it is compulsory to reduce the size of these vectors to minimize the computational burden of the problem while maintaining accuracy. To this end only 20 components of such vectors were retained, a number that was found to satisfy both suitable accuracy and limited computational burden. Index $i=1,2, \ldots, 20$ refers to the 20 discretized frequency points used to reproduce the frequency response of each impedance (see the red points in Fig. 7). Note that $X$ is the reactance, $X=2 \cdot \pi \cdot f \cdot L$ (longitudinal impedance) or $X=1 /(2 \cdot \pi \cdot f \cdot C$ ) (transversal impedance).

It is noted that when analyzing the curves defining the longitudinal parameters $R_{l}\left(f_{i}\right)$ and $L_{l}\left(f_{i}\right)$ with $i=$ $1,2, \ldots 20$, the values of the $\left(R_{1, l}, R_{2, l}, R_{3, l}, R_{4, l}, R_{5, l}, L_{1, l}, L_{2, l}, L_{3, l}, L_{4, l}, L_{5, l}\right)$ parameters are changed at each internal iteration of the GA process to find out the optimal set of values minimizing the objective function defined 
by (3). The values of these parameters are coded into binary strings constituting the chromosomes and are changed according to the GA rules so that $R_{l}=R_{c a l}$ and $X_{l}=2 \cdot \pi \cdot f \cdot L_{l}=X_{c a l}$ are calculated according to the real and imaginary parts of the equivalent impedance of the ladder circuit shown in Fig. 3. The elitist scattered crossover operation is applied, producing a random binary vector at each generation from which the genes of the two parents are combined to generate an offspring. Next, the mutation operation is applied, by which the GA creates random variations in the chromosomes information, thus producing genetic diversity and enabling GA to search for wider spaces. The whole process is repeated until achieving a preestablished tolerance threshold.

However, after a thorough study, it was noticed that model performance can be further optimized by iteratively applying the above-explained process. To this end this paper proposes adding an additional iteration loop to the GA process which allows reducing the error in the objective function and thus model accuracy. In this iterative process, the GA solution attained at each iteration step is the initial condition or seed value for the initial population of the subsequent iteration as shown in Fig. 6. 


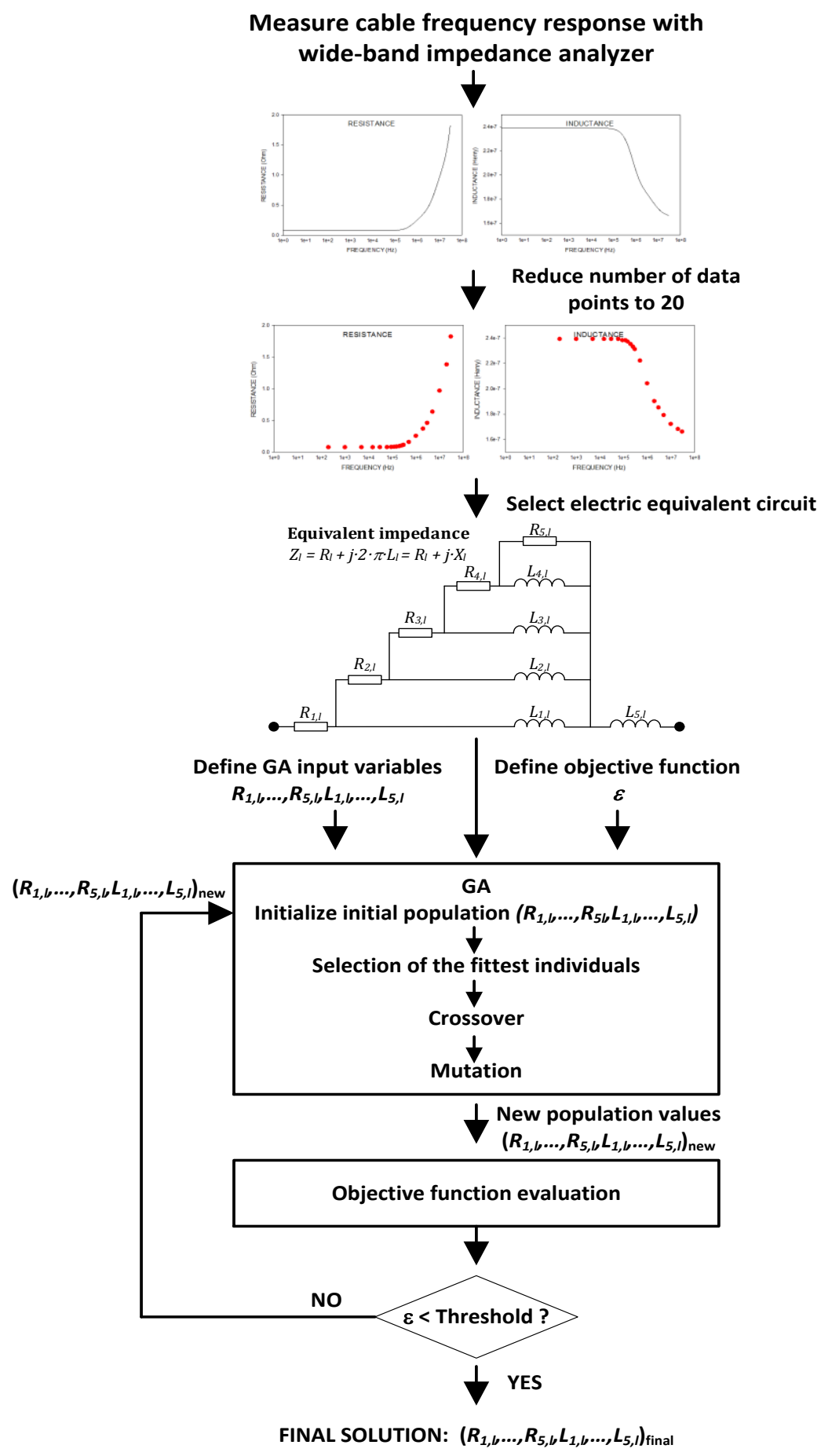

Fig. 6. Proposed IGA-based approach to adjust the longitudinal $\left(R_{i, l}, L_{i, l}\right)$ parameters. The same process is applied to adjust the transversal conductor-conductor and conductors-screen parameters, $\left(G_{i, t, c c}, C_{i, t, c c}\right)$ and $\left(G_{i, t, c s}, C_{i, t, c s}\right)$, respectively.

The same process is applied to impedances $Z_{t, c c}$ and $Z_{t, c s}$ to determine, respectively, the optimal values of the parameters $\left(R_{1, t, c c}, R_{2, t, c c}, R_{3, t, c c}, R_{4, t, c c}, C_{1, t, c c}, C_{2, t, c c}, C_{3, t, c c}, C_{4, t, c c}\right)$ and $\left(R_{1, t, c s}, R_{2, t, c s}, R_{3, t, c s}, R_{4, t, c s}, C_{1, t, c s}, C_{2, t, c s}, C_{3, t, c s}, C_{4, t, c s}\right)$ that minimize the objective function described by (3). 
The optimization process carried out in this work has been implemented in the MATLAB $®$ R2014a Optimization Toolbox.

\section{EXPERIMENTAL RESULTS}

Different experimental results were carried out with the aim of adjusting the values of the parameters of the cell model and validating the cable model, as detailed in the following subsections.

\subsection{Adjusted parameter values of the identical cells}

A three-wire cable $\left(3 \times 6 \mathrm{~mm}^{2}\right)$ with a copper braid with reference EXZHELLENT RC4Z1-K (AS) 0.6/1

$\mathrm{kV}$ from GeneralCable ${ }^{\circledR}$ was used to experimentally validate the cable model. The experimental tests were carried at the facilities of the Universitat Politècnica de Catalunya (UPC), using an Agilent 4294A wideband high-precision impedance analyzer. The Agilent 4294A provides hundreds of parameter-frequency data points for a full scanning within the $40 \mathrm{~Hz}-110 \mathrm{MHz}$ frequency interval.

Two cable samples of lengths $0.1 \mathrm{~m}$ and $1 \mathrm{~m}$ were used to generate the single-cell model $(1$ cell $=1 \mathrm{~m}$ of cable) from experimental data. With the aim of reducing the computational requirements and speeding up the IGA-based process to select the optimal parameters' values of the longitudinal and transversal impedances shown in Figs. 3, only 20 points were retained as shown in Figs. 7. It is noted that data shown in Figs. 7 have been obtained by applying the Steps 1-3 in Fig. 5.
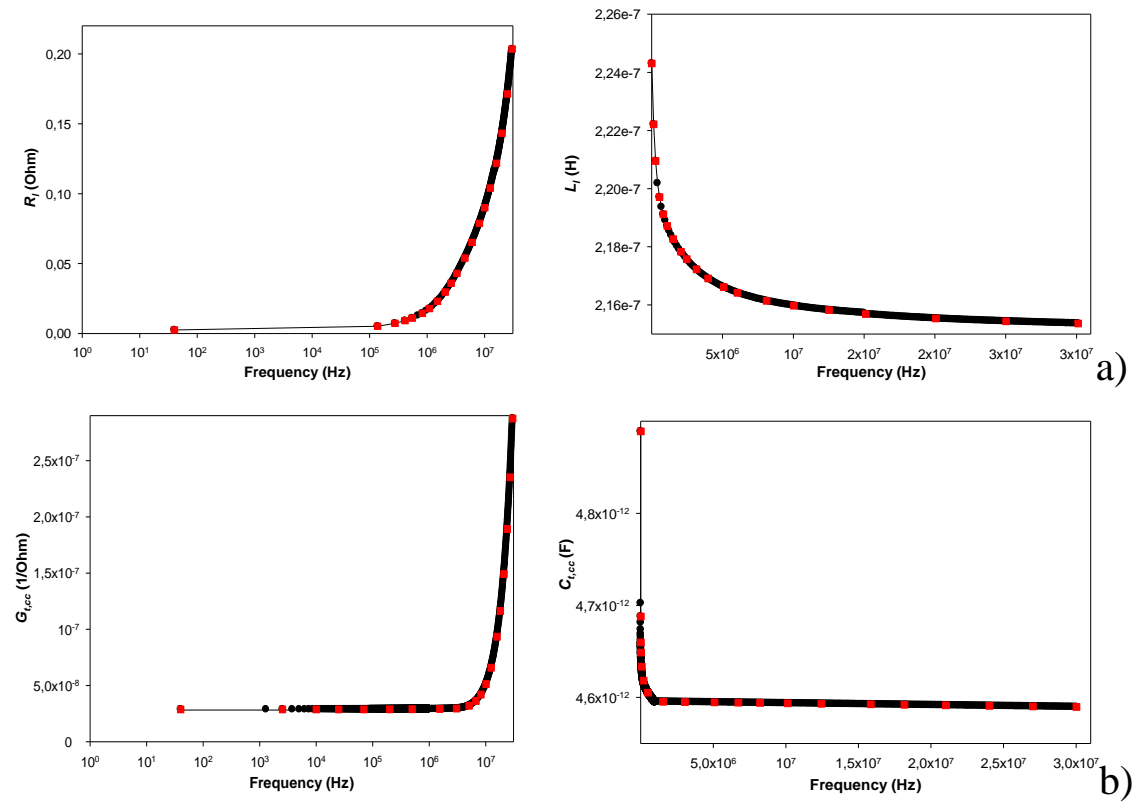

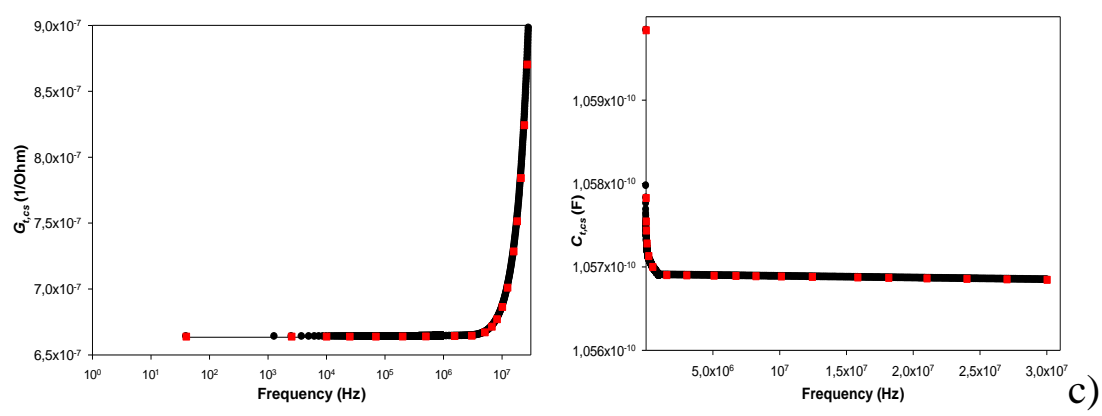

Fig. 7. Three-wire screened cable with a length of $1 \mathrm{~m}$. Values from experimental data of the cell parameters $R_{l}, L_{l}, C_{t, c c}, C_{t, c s}, G_{t, c c}$ and $G_{t, c s}$ in the $40 \mathrm{~Hz}-30 \mathrm{MHz}$ frequency range obtained by solving (2) and applying the steps $1-3$ in Fig. 5. The red points indicate the values retained in the curves for the posterior IGA process. a) Longitudinal parameters $R_{l}$ and $L_{l}$. b) Conductor-conductor transversal parameters $G_{t, c c}$ and $C_{t, c c}$. c) Conductor-screen transversal parameters $G_{t, c s}$ and $C_{t, c s}$.

The next step is to determine the parameters' values of the single-cell model from the experimental frequency responses summarized in Fig. 7. The values obtained after applying the IGA optimization approach are summarized in Tables II, III and IV, which are used in the next sections to validate the cable model.

TABLE II. PARAMETERS OF THE LONGITUDINAL IMPEDANCE MODEL OBTAINED THROUGH IGA OPTIMIZATION (FIG. 3A)

\begin{tabular}{cc}
\hline Cable parameter & Value \\
\hline$R_{1, l}$ & $3.30 \mathrm{~m} \Omega$ \\
$R_{2, l}$ & $35.96 \mathrm{~m} \Omega$ \\
$R_{3, l}$ & $252.7 \mathrm{~m} \Omega$ \\
$R_{4, l}$ & $981.0 \mathrm{~m} \Omega$ \\
$R_{5, l}$ & $5.180 \Omega$ \\
$L_{1, l}$ & $13.88 \mathrm{nH}$ \\
$L_{2, l}$ & $18.39 \mathrm{nH}$ \\
$L_{3, l}$ & $15.72 \mathrm{nH}$ \\
$L_{4, l}$ & $19.52 \mathrm{nH}$ \\
$L_{5, l}$ & $210.70 \mathrm{nH}$ \\
\hline
\end{tabular}

TABLE III. PARAMETERS OF THE CONDUCTOR-

CONDUCTOR TRANSVERSAL IMPEDANCE MODEL OBTAINED THROUGH IGA OPTIMIZATION (FIG. 3A)

\begin{tabular}{cc}
\hline Cable parameter & Value \\
\hline$R_{1, t, c c}$ & $13.35 \mathrm{k} \Omega$ \\
$R_{2, t, c c}$ & $3.531 \mathrm{M} \Omega$ \\
$R_{3, t, c c}$ & $14.34 \Omega$ \\
$R_{4, t, c c}$ & $1.630 \Omega$ \\
$C_{1, t, c c}$ & $3.476 \mathrm{pF}$ \\
$C_{2, t, c c}$ & $0.890 \mathrm{pF}$ \\
$C_{3, t, c c}$ & $2.269 \mathrm{pF}$ \\
$C_{4, t, c c}$ & $0.500 \mathrm{pF}$ \\
\hline
\end{tabular}


TABLE IV. PARAMETERS OF THE CONDUCTOR-

SCREEN TRANSVERSAL IMPEDANCE MODEL

OBTAINED THROUGH IGA OPTIMIZATION (FIG. 3A)

\begin{tabular}{cc}
\hline Cable parameter & Value \\
\hline$R_{1, t, c s}$ & $178.1 \mathrm{k} \Omega$ \\
$R_{2, t, c s}$ & $1.175 \mathrm{k} \Omega$ \\
$R_{3, t, c s}$ & $35.51 \mathrm{p} \Omega$ \\
$R_{4, t, c s}$ & $0.644 \mathrm{p} \Omega$ \\
$C_{1, t, c s}$ & $123.90 \mathrm{nF}$ \\
$C_{2, t, c s}$ & $7.327 \mathrm{pF}$ \\
$C_{3, t, c s}$ & $1.083 \mathrm{pF}$ \\
$C_{4, t, c s}$ & $6.956 \mathrm{pF}$ \\
\hline
\end{tabular}

\subsection{Experimental validation of the frequency response of a $5 \mathbf{m}$ length cable model}

A third cable sample of $5 \mathrm{~m}$ length was analyzed in order to validate the proposed system. A five-cell model was generated to simulate the frequency response of the $5 \mathrm{~m}$ cable from the single-cell model shown in Fig. 3 and the values of the cell parameters adjusted via IGA summarized in Tables II, III and IV. This model was exported to the EMTPWorks® 2015a(3.1.0) transients simulation software [35], which is shown in Fig. 8.
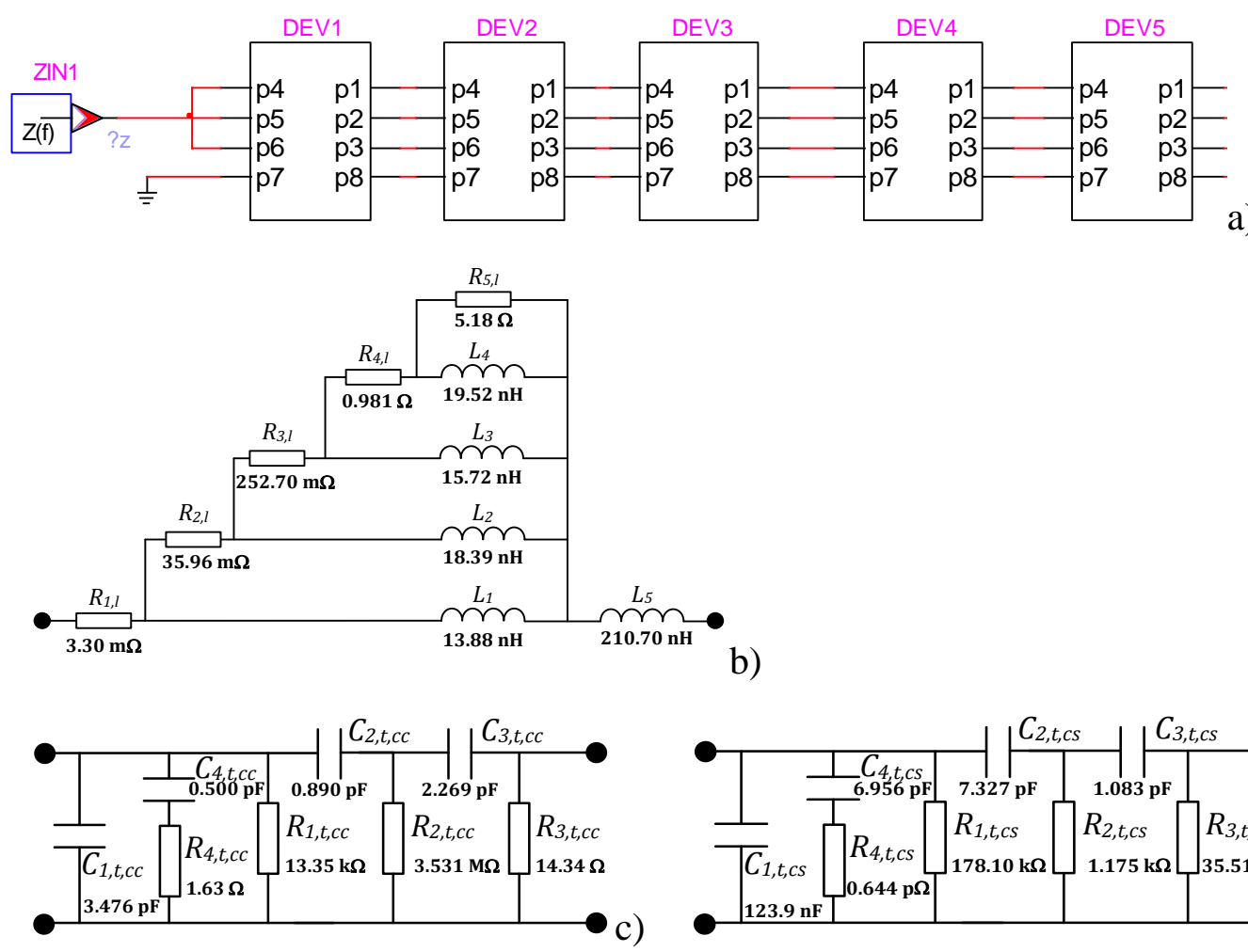

b)

Fig. 8. a) Five-cells cable model to simulate the behavior of a $5 \mathrm{~m}$ cable length. b) Conductor-conductor transversal impedance of each cell. c). d) Conductor-screen transversal impedance of each cell.

The results of the four experimental tests performed to the $5 \mathrm{~m}$ cable (two short circuit tests SC2 and SC3 and the two open circuit tests OC2 and OC3) using the Agilent 4294A impedance analyzer in the $40 \mathrm{~Hz}-$ 
$30 \mathrm{MHz}$ frequency interval and results from simulations carried out with the EMTPWorks@ 2015a(3.1.0) package according to the model presented in Figs. 8 are compared in Figs. 9.
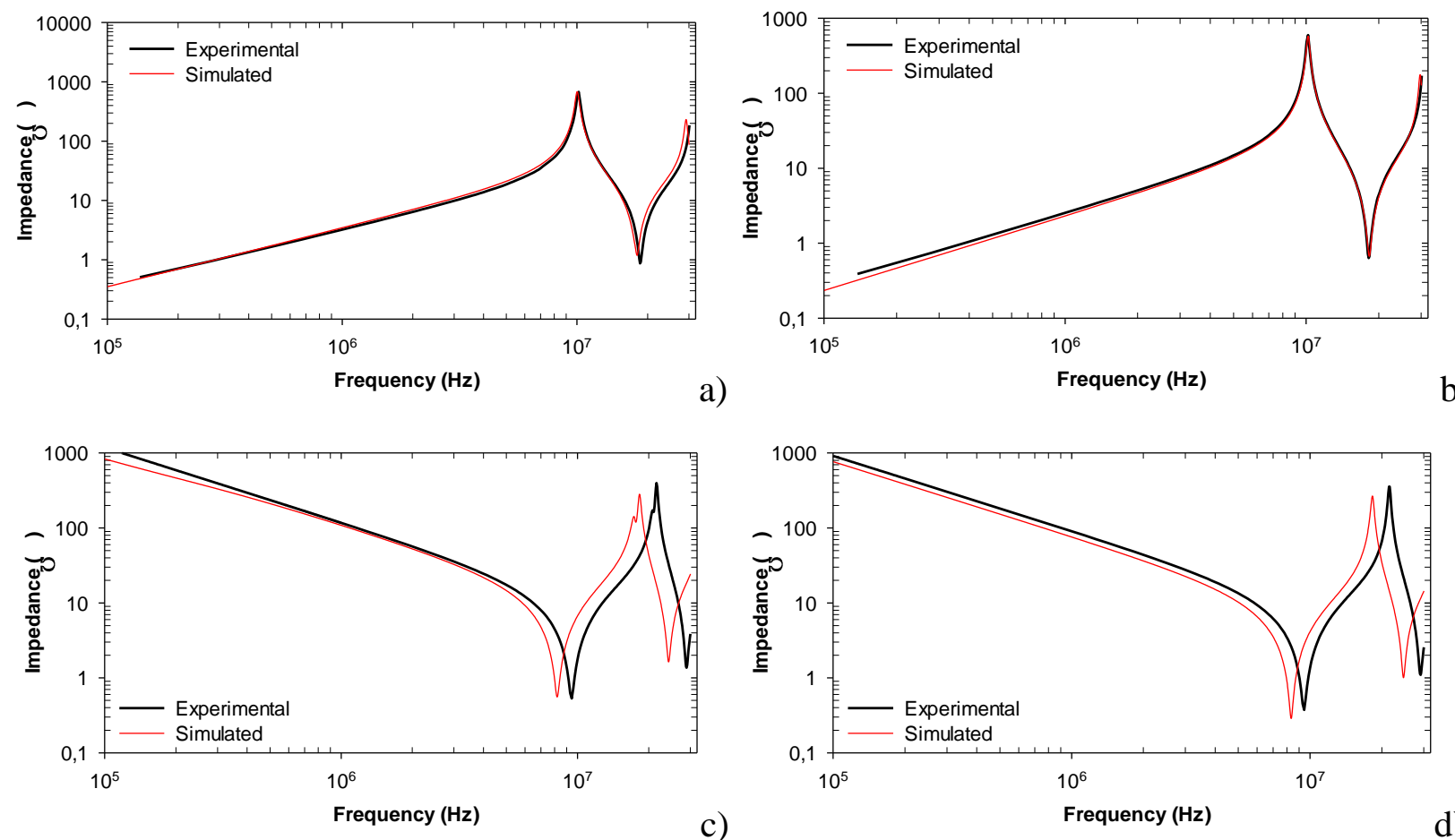

Fig. 9. Comparison between simulated and experimental frequency responses of the four tests applied to the three-wire screened cable with a length of $5 \mathrm{~m}$. a) Impedance-frequency graph corresponding to SC2. b) Impedance-frequency graph corresponding to SC3. c) Impedance-frequency graph corresponding to OC2. d) Impedance-frequency graph corresponding to OC3.

Results presented in Figs. 9 show a good agreement between the measured frequency response and the simulated one according to the model whose parameters were adjusted by means of the IGA-based method presented in this work.

\subsection{Experimental validation of the temporal response of a 30 m length cable model}

To validate the accuracy of the proposed simulation method, a fourth cable sample of $30 \mathrm{~m}$ length was analyzed in order to magnify the magnitude of the overvoltage induced at the load side of the cables. In this case a similar model to that depicted in Figs. 8 but with 30 cells was built and simulated with the EMTPWorks® 2015a(3.1.0) package.

A first open circuit test consisting in applying a $60 \mathrm{~V}_{\mathrm{dc}}$ step voltage to conductor \#1 (conductors \#2, \#3 and the screen are connected to the negative terminal of the DC voltage source) of the $30 \mathrm{~m}$ cable configuration, and measuring the voltage at the load side of conductor \#1 as indicated in Fig. 9, was carried out, whose results are shown in Fig. 10. 


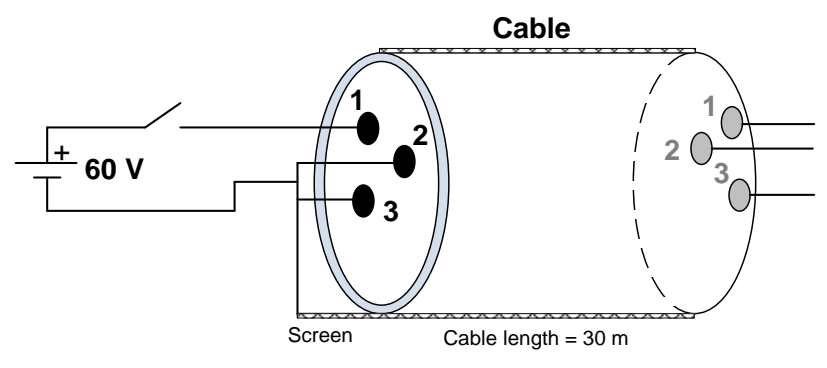

a)

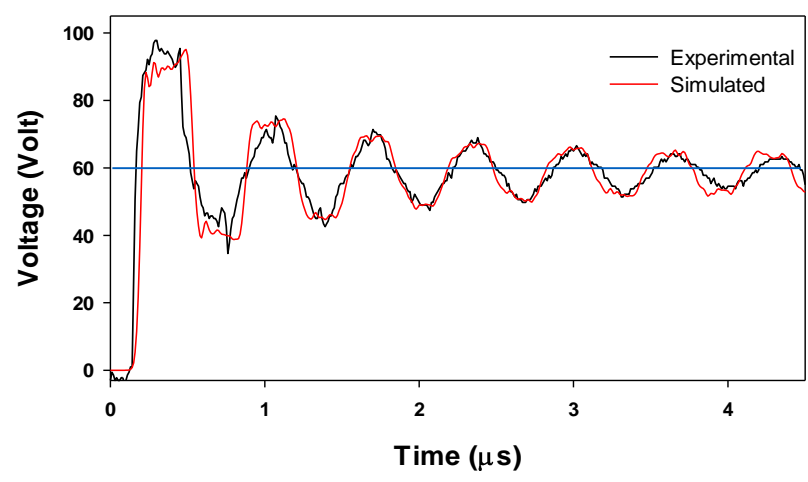

b)

Fig. 10. Open circuit test with a cable length of $30 \mathrm{~m}$. Transient temporal response to a step voltage. a) Setup to determine the transient response to a $60 \mathrm{~V}_{\mathrm{dc}}$ step voltage applied to conductor \#1, whereas conductors \#2, \#3 and the screen are connected to the negative terminal of the DC voltage source. b) Simulated versus experimental voltages at the load side of the energized conductor (conductor \#1).

Results presented in Fig. 10b show a great similarity between experimental and simulated results, thus proving the accuracy and usefulness of the proposed approach.

A second open circuit test was conducted by using a three-phase PWM-modulated inverter to feed the three-wire screened cable of $30 \mathrm{~m}$ length, as illustrated in Figs. 11. The input DC voltage was $360 \mathrm{~V}$, the switching frequency $20 \mathrm{kHz}$, the AC output frequency $400 \mathrm{~Hz}$ and the modulation index 0.9 .

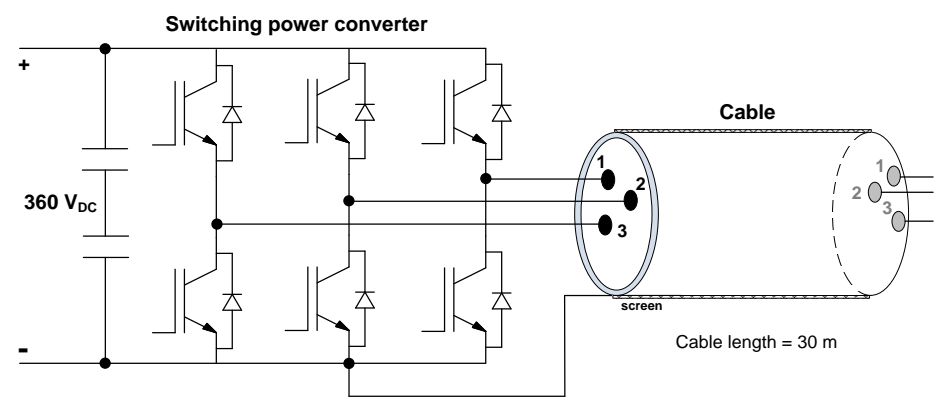




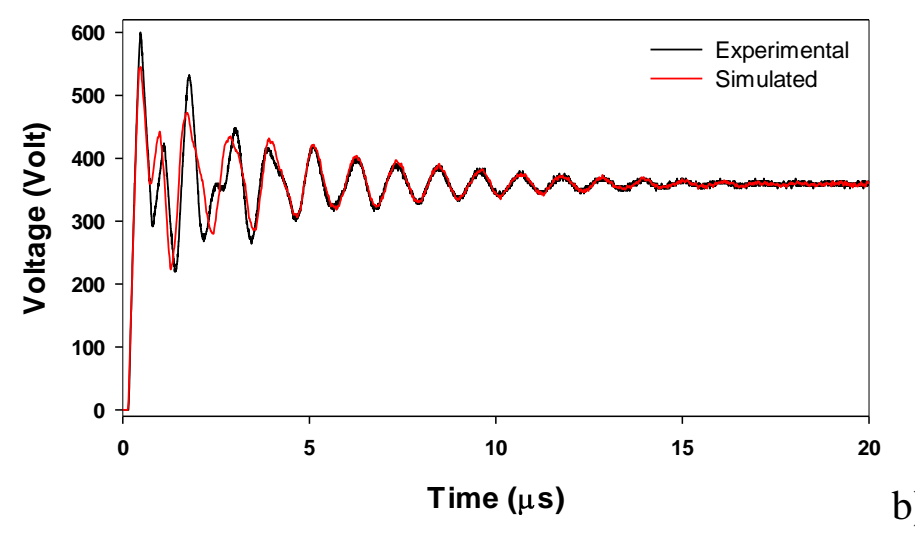

Fig. 11. Open circuit test with a cable length of $30 \mathrm{~m}$. Cable response to a three-phase PWM modulated $400 \mathrm{~Hz}$ voltage signal. a) Experimental setup used to measure the output voltage at the load side of the energized conductor (conductor \#1). b) Simulated versus experimental voltages at the load side of the energized conductor (conductor \#1).

It is worth noting that the errors between experimental and simulation results have different origins. There is an inherent error due to the assumptions and simplifications made in the lumped-parameter model dealt with which cannot be avoided by applying any fitting technique. Experimental data from the short circuit and open circuit tests may contain noise and interferences which also contribute to the model's inaccuracy. And finally, the parameter fitting process itself may also contribute to the discrepancies between experimental and simulated results.

The computational burden of the proposed model as a function of the number of series-connected cells once adjusted the cell parameters is summarized in Table V.

\begin{tabular}{ccc}
\multicolumn{3}{c}{ TABLE V. COMPUTATIONAL TIME REQUIRED TO } \\
SIMULATE 1 MICRO-SECOND WITH A TIME-STEP \\
OF 0.1 NANO-SECOND (30 METER CABLE) \\
\hline Cells number & $\begin{array}{c}\text { Computation } \\
\text { time } \\
\text { (seconds) }\end{array}$ & $\begin{array}{c}\text { Relative } \\
\text { time }\end{array}$ \\
\hline 1 & 0.56160 & 1.000 \\
5 & 0.79561 & 1.417 \\
10 & 0.90481 & 1.611 \\
20 & 1.18561 & 2.111 \\
30 & 1.60681 & 2.861 \\
50 & 2.35562 & 4.195 \\
100 & 4.57083 & 8.139 \\
\hline
\end{tabular}

Results summarized in Table V are based on an Intel ${ }^{\circledR}$ Core ${ }^{\mathrm{TM}}$ i7-2600 - 3.40GHz, 8.00 GBytes RAM computer. It is noted that the proposed modelling approach is much faster than the finite element method (FEM) alternative [36] since a 3D-FEM model is required because of the finite length of the cable and a very fine mesh is needed due to the analyzed frequency interval. 


\section{CONCLUSION}

Accurate high-frequency cable models are appealing due to the increasing use of electronic power converters in different areas including industry, aeronautics or automobile among others. It is well known that transient overvoltage phenomena can appear, whose impact depends on cable length and configuration and switching frequency. Ladder network models can be used to precisely replicate the wide-band frequency response of such a system. These models include a rather large number of $R, L$ and $C$ components, whose values have be adjusted to match the simulated response with the experimental one. This paper has presented an improved optimization GA-based method to fit the parameters of a ladder lumped parameter multi-cell high-frequency model of a multi-wire screened power cable. The iterative GA-based approach presented in this paper allows improving the accuracy of the solution attained while limiting the computational requirements. Experimental overvoltage data acquired from different cable samples have validated the usefulness of the proposed approach. Although the IGA-based approach has been applied to adjust the parameters of multi-wire cable models, it can also be extended to other applications such to determine the optimal parameter values of filters or high-frequency models of electrical machines such as motors, generators or transformers.

\section{ACKNOWLEDGMENT}

The authors gratefully acknowledge the technical support provided by Powersys Inc. with the EMTP-RV simulation software. This work was partially supported by the Spanish Ministry of Science and Technology [TRA2013-46757-R] and the European Commission [Clean Sky program, FPT-JT MultyCaB 632458].

\section{REFERENCES}

[1] Stevanovic I, Wunsch B, Madonna GL, Skibin S. High-Frequency Behavioral Multiconductor Cable Modeling for EMI Simulations in Power Electronics. IEEE Trans Ind Informatics 2014;10:1392400. doi:10.1109/TII.2014.2307198.

[2] Bulington EJ, Abney S, Skibinski GL. Cable alternatives for PWM AC drive applications. Ind. Appl. Soc. 46th Annu. Pet. Chem. Tech. Conf. (Cat.No. 99CH37000), IEEE; n.d., p. 247-59. doi:10.1109/PCICON.1999.806443.

[3] Bartolucci EJ, Finke BH. Cable design for PWM variable-speed AC drives. IEEE Trans Ind Appl 2001;37:415-22. doi:10.1109/28.913704.

[4] Wu X, Zhang H, Karady GG. Transient analysis of inductive induced voltage between power line and nearby pipeline. Int J Electr Power Energy Syst 2017;84:47-54. doi:10.1016/j.ijepes.2016.04.051.

[5] Sabatier J, Youssef T, Pellet M. An HVDC line parameters estimation method without optimization. vol. 83. 2016. doi:10.1016/j.ijepes.2016.04.001.

[6] Torrez Caballero P, Marques Costa EC, Kurokawa S. Frequency-dependent line model in the time domain for simulation of fast and impulsive transients. Int J Electr Power Energy Syst 2016;80:17989. doi:10.1016/j.ijepes.2016.01.051.

[7] Rodriguez Valdez C, Tallam RM, Kerkman RJ. A new frequency-dependent-model for cables. 2008 
IEEE Int. Conf. Electro/Information Technol., IEEE; 2008, p. 151-8. doi:10.1109/EIT.2008.4554285.

[8] Wang L, Ngai-Man Ho C, Canales F, Jatskevich J. High-Frequency Modeling of the Long-CableFed Induction Motor Drive System Using TLM Approach for Predicting Overvoltage Transients. IEEE Trans Power Electron 2010;25:2653-64. doi:10.1109/TPEL.2010.2047027.

[9] Wang L, Ho CNM, Canales F, Jatskevich J. High-frequency cable and motor modeling of longcable-fed induction motor drive systems. 2010 IEEE Energy Convers. Congr. Expo., IEEE; 2010, p. 846-52. doi:10.1109/ECCE.2010.5617906.

[10] de Paula H, Chaves MLR, Andrade DA, Freitas MAA, Domingos JL. A Simple and Accurate Cable Modeling Suitable for High-frequency Phenomena Analysis in PWM Motor Drives. IEEE 36th Conf. Power Electron. Spec. 2005., IEEE; 2005, p. 680-6. doi:10.1109/PESC.2005.1581700.

[11] De Paula H, de Andrade DA, Chaves MLR, Domingos JL, de Freitas MAA. Methodology for Cable Modeling and Simulation for High-Frequency Phenomena Studies in PWM Motor Drives. IEEE Trans Power Electron 2008;23:744-52. doi:10.1109/TPEL.2007.915759.

[12] Dommel H. Digital Computer Solution of Electromagnetic Transients in Single-and Multiphase Networks. IEEE Trans Power Appar Syst 1969;PAS-88:388-99. doi:10.1109/TPAS.1969.292459.

[13] Dhaene T, de Zutter D. Selection of lumped element models for coupled lossy transmission lines. IEEE Trans Comput Des Integr Circuits Syst 1992;11:805-15. doi:10.1109/43.144845.

[14] Moreira AF, Lipo TA, Venkataramanan G, Bernet S. High-frequency modeling for cable and induction motor overvoltage studies in long cable drives. IEEE Trans Ind Appl 2002;38:1297-306. doi:10.1109/TIA.2002.802920.

[15] Eslamian; M, Vahidi; B, Hosseinian SH. Combined analytical and FEM methods for parameters calculation of detailed model for dry-type transformer. Simul Model Pract Theory 2010;18:390-403. doi:0.1016/j.simpat.2009.12.005.

[16] Shabestary MM, Ghanizadeh AJ, Gharehpetian GB, Agha-Mirsalim M. Ladder Network Parameters Determination Considering Nondominant Resonances of the Transformer Winding. IEEE Trans Power Deliv 2014;29:108-17. doi:10.1109/TPWRD.2013.2278784.

[17] Mohammed OA, Ganu S. FE-Circuit Coupled Model of Electric Machines for Simulation and Evaluation of EMI Issues in Motor Drives. IEEE Trans Magn 2010;46:3389-92. doi:10.1109/TMAG.2010.2045746.

[18] Groling C, Amlang B, Schumacher W, Grobe M. High-frequency lumped parameter winding model of a synchronous servo drive. 2008 IEEE Power Electron. Spec. Conf., IEEE; 2008, p. 2118-24. doi:10.1109/PESC.2008.4592256.

[19] Mohammed OA, Ganu S, Abed N, Liu S, Liu Z. High frequency PM synchronous motor model determined by FE analysis. IEEE Trans Magn 2006;42:1291-4. doi:10.1109/TMAG.2006.872412.

[20] Hui SYR, Christopoulos C. Computer simulation of a converter-fed DC drive using the transmission-line modeling technique. IEEE Trans Power Electron 1991;6:636-44. doi:10.1109/63.97763.

[21] Johns PB, O'Brien M. Use of the transmission-line modelling (t.1.m.) method to solve non-linear lumped networks. Radio Electron Eng 1980;50:59. doi:10.1049/ree.1980.0006.

[22] Weens Y, Idir N, Franchaud JJ, Bausiére R. HF Model of an Unshielded 2-Wire Energy Cable Submitted to Power Electronic Converter Constraints. Int J Model Simul 2015.

[23] Lin JT, Chen C-M. Simulation optimization approach for hybrid flow shop scheduling problem in semiconductor back-end manufacturing. Simul Model Pract Theory 2015;51:100-14. doi:10.1016/j.simpat.2014.10.008.

[24] Kitayama S, Natsume S. Multi-objective optimization of volume shrinkage and clamping force for plastic injection molding via sequential approximate optimization. Simul Model Pract Theory 2014;48:35-44. doi:10.1016/j.simpat.2014.07.004.

[25] Akbal B, Urkmez A. Genetic algorithm to solve electrical network problems. 2013 Int. Conf. Electron. Comput. Comput., IEEE; 2013, p. 235-8. doi:10.1109/ICECCO.2013.6718272. 
[26] Shintemirov A, Tang WH, Wu QH. Transformer Core Parameter Identification Using Frequency Response Analysis. IEEE Trans Magn 2010;46:141-9. doi:10.1109/TMAG.2009.2026423.

[27] Samarawickrama K, Jacob ND, Gole AM, Kordi B. Impulse Generator Optimum Setup for Transient Testing of Transformers Using Frequency-Response Analysis and Genetic Algorithm. IEEE Trans Power Deliv 2015;30:1949-57. doi:10.1109/TPWRD.2015.2429554.

[28] Loyka SL. Applying genetic algorithm to modeling nonlinear transfer functions. 4th Int. Conf. Telecommun. Mod. Satell. Cable Broadcast. Serv. TELSIKS’99 (Cat. No.99EX365), vol. 1, IEEE; 1999, p. 247-50. doi:10.1109/TELSKS.1999.804737.

[29] Sales C, Rodrigues RM, Lindqvist F, Costa J, Klautau A, Ericson K, et al. Line Topology Identification Using Multiobjective Evolutionary Computation. IEEE Trans Instrum Meas 2010;59:715-29. doi:10.1109/TIM.2009.2025991.

[30] Kwon C, Sudhoff SD. Genetic Algorithm-Based Induction Machine Characterization Procedure With Application to Maximum Torque Per Amp Control. IEEE Trans Energy Convers 2006;21:40515. doi:10.1109/TEC.2006.874224.

[31] Weens Y, Idir N, Franchaud JJ, Bausiere R. High frequency model of a shielded 4-wire energy cable. 2005 Eur. Conf. Power Electron. Appl., IEEE; 2005, p. 10 pp.-pp.P.10. doi:10.1109/EPE.2005.219644.

[32] Kaiser KL. Electromagnetic Compatibility Handbook. CRC Press; 2004.

[33] Moreau M. Modélisation haute fréquence des convertisseurs d'énergie : application à l'étude des émissions conduites vers le réseau 2009:167.

[34] H. Saavedra, Riba J-R, Romeral L. Multi-objective Optimal Design of a Five-Phase Fault-Tolerant Axial Flux PM Motor. Adv Electr Comput Eng 2015;15:69-76. doi:10.4316/AECE.2015.01010.

[35] J., Mahseredjian, Dewhurst C. EMTP-RV User Manual. Version 3.3. Le Puy-Sainte-Réparade, France: 2016.

[36] Hio Nam O, Blackburn TR, Phung BT. Modeling propagation characteristics of power cables with finite element techniques and ATP. 2007 Australas. Univ. Power Eng. Conf., IEEE; 2007, p. 1-5. doi:10.1109/AUPEC.2007.4548087. 